\title{
A Giant Frontal Meningioma with Psychiatric Symptoms in a Young Woman: A Case Report
}

\author{
Thi Phuong Hoai Dinh ${ }^{1,2^{*}}$, Dang Thi Mai ${ }^{1 *}$, Thi Mai Dieu Tran ${ }^{1 *}$, Hai Duong Pham ${ }^{1 \sharp}$, Vinh Lac Nguyen ${ }^{1 \#}$, Thanh \\ Minh Nguyen ${ }^{1 \#}$
}

${ }^{1}$ Hue University of Medicine and Pharmacy, Hue University, Hue, Vietnam

${ }^{2}$ Online Research Club (http://www.onlineresearchclub.org/)

\begin{abstract}
Background:Frontal meningioma is often asymptomatic but patients might experience psychiatric symptoms. Since symptoms are atypical in giant meningioma, to the best of our knowledge, we present a rare clinical scenario.

Case Presentation: A 24-year old female patient with no medical history presented with two months of depressive symptoms, personality changes such as easy irritability, and feelings of frustration and mental depression, as well as forgetfulness and blurred vision. Brain magnetic resonance imaging (MRI) revealed a well-defined markedly enhancing lesion in the left frontal region measuring $65 \mathrm{x}$ $70 \times 70 \mathrm{~mm}$ with mass effects to the adjacent brain tissue and associated surrounding edema which was pressed to the frontal horn of the lateral ventricle. The patient underwent frontal unilateral craniotomy and excision of the tumor. After surgery, the quality of life was improved.

Conclusion: Meningioma with psychiatric symptoms is quite rare. This clinical case helps clinicians avoid missing symptoms and use appropriate management strategies for patients with giant meningiomas.

Keywords: Atypical psychiatric symptoms; Giant meningioma; Unilateral frontal craniotomy.
\end{abstract}

*Correspondence to

Dinh Thi Phuong Hoai, Hue University of Medicine and Pharmacy, Hue University, Hue, Vietnam.

Email: phuonghoai1412.md@ gmail.com

\#Authors equally contributed to the work.

Published online March 27 2021

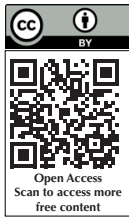

Citation: Dinh TPH, Mai DT, Tran TMD, Pham HD, Nguyen VL, Nguyen TM. A Giant Frontal Meningioma with Psychiatric Symptoms in a Young Woman: A Case Report. Clin Neurosci J. 2021;8(2):99-102. doi:10.34172/icnj.2021.21.

\section{Introduction}

Meningioma is a common benign idiopathic extra-axial tumor arising in the outer layer of the leptomeninges from arachnoid cap cells. ${ }^{1}$ Primary or metastasis brain tumors typically lead to the development of focal neurological deficits, such as hemiparesis, sensory deficiency, and aphasia. However, the external compression of the frontal lobes by meningioma does not cause any symptoms other than gradual changes in personality and comprehension until they are substantially enlarged, leading to its recognized diagnosis as a "silent tumor".

Meningiomas, as the most commonly seen intracranial tumors, account for approximately 13\%-26\% of intracranial tumors. Most of them are slow-growing, and many are parenthetically defined. ${ }^{3}$ While the etiology of meningioma is unclear, researchers believe that these tumors occur irregularly and may be associated with neurofibromatosis 2 (NF2), meningioangiomatosis (MA) and Gorlin syndrome as a family syndrome. ${ }^{1,4}$

The clinical symptoms are typically based on the anatomical location. ${ }^{5}$ We present a woman with depression and changes in personality, which displayed spaceoccupying lesions on further examination, indicating a giant frontal meningioma which we successfully removed.

\section{Case Presentation}

A 24-year-old woman without a prior medical and psychological history was referred for inappropriate activity to a psychiatric clinic. She mainly had signs of depression, changes in personality, and was easily irritable, frustrated, and mentally stressed. With low memory and blurred vision, she had been forgetful as well. She had no history of head trauma, neurological focal deficit, or mental illness. She never consumed or smoked alcohol and did not use any illicit drugs.

She was found to be time, place, and person-oriented on examination but had low attention and focus with poor judgments. Her score on the Mini-Mental State Examination score was $25 / 30$. With a pulse rate of 70 beats/min, her blood pressure was 120/70 mm Hg. Fundoscopy did not show pink papillae, or any border or hemorrhage. Neurological investigations were all unremarkable, including cranial nerve and other systemic examinations. The results of her blood test were normal. A brain magnetic resonance imaging (MRI) was requested, and the results showed a well-defined significantly enhancing lesion in the left frontal region measuring $65 \times$ $70 \times 70 \mathrm{~mm}$, with a fairly even edge, compression on the adjacent brain parenchyma with an associated significant

(C) 2021 The Author(s). This is an open access article distributed under the terms of the Creative Commons Attribution License (http:// creativecommons.org/licenses/by/4.0/), which permits unrestricted use, distribution, and reproduction in any medium, provided the original work is properly cited. 
surrounding edema. The highly contrast-absorbing mass was quite homogeneous after injection, and contained a necrotic material that was not contrast-absorbing. The mass pushed on both sides of the frontal horn of the ventricle, moving the middle line by $23 \mathrm{~mm}$. These characteristics are consistent with a giant left frontal meningioma (Figure 1). Unilateral frontal craniotomy and tumor excision was done for the patients.

The purpose of the patient's operation was complete tumor resection with maximum function preservation of the regions of the brain related to insight. The patient was placed in a supine position during surgery with her head securely fixed with the Mayfield frame. Her hair was shaved after general anesthesia and the location of the skin incision site was marked (Figure 1), followed by povidoneiodine draping. A skin incision was made. Initial bone drilling and osteotomy were performed. Tack-up sutures were laid down and opened up the dura. The tumor was seen attached to the dura and we accomplished complete tumor resection after thorough debulking of the tumor in a piece by piece manner. The complete hemostasis was secured and the bone flap replaced with plates and screws. The drain tube was put in place and the skin closed in layers. The patient was admitted to the intensive care unit for neurosurgical therapy. Postoperative brain MRI indicated complete resection of the tumor (Figure 2).

Grade I meningioma as classified by the World Health Organization on pathology (Figure 3). The patient was referred to the neuropsychology clinic for a

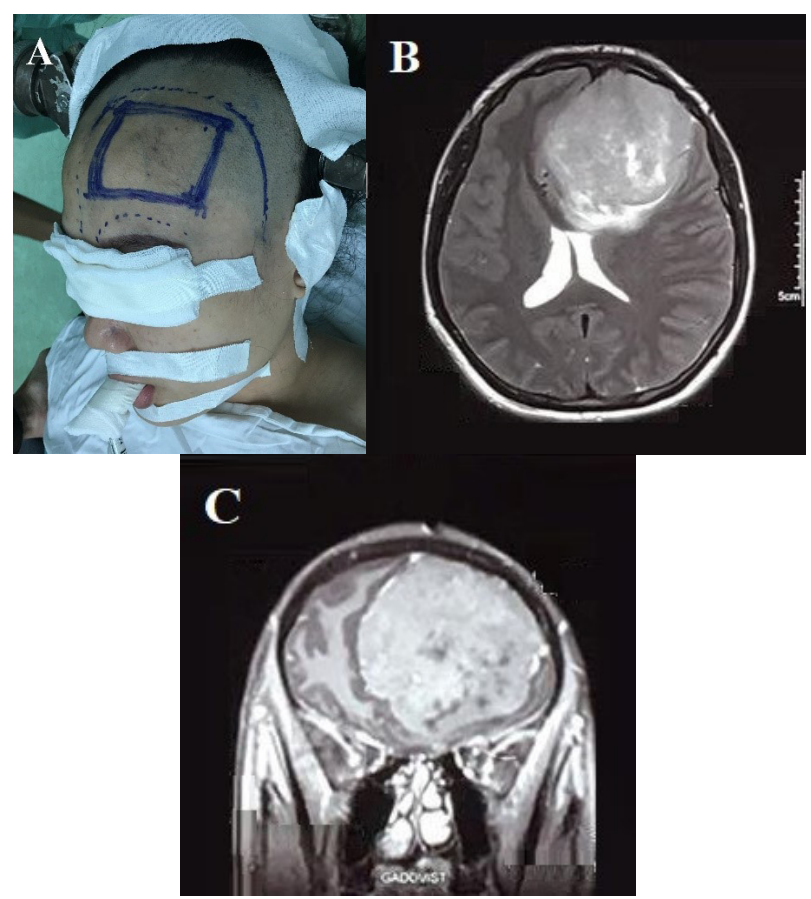

Figure 1. (A) Preparing for the removal of left frontal meningioma by microsurgery before surgery. (B) (axial) - (C) (coronal). The mass presses both sides of the frontal horn of the lateral ventricle, the middle line has shifted $23 \mathrm{~mm}$.

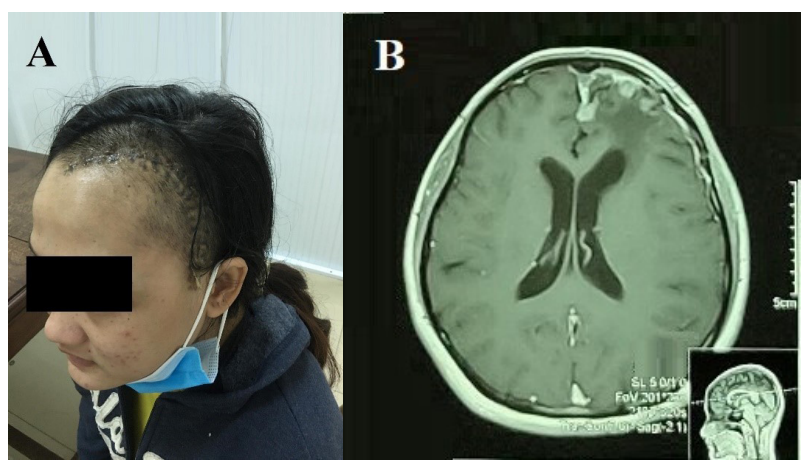

Figure 2. (A) Patient re-examination after 1 month of surgery. (B) Postoperative MRI-brain indicated complete resection of tumor.

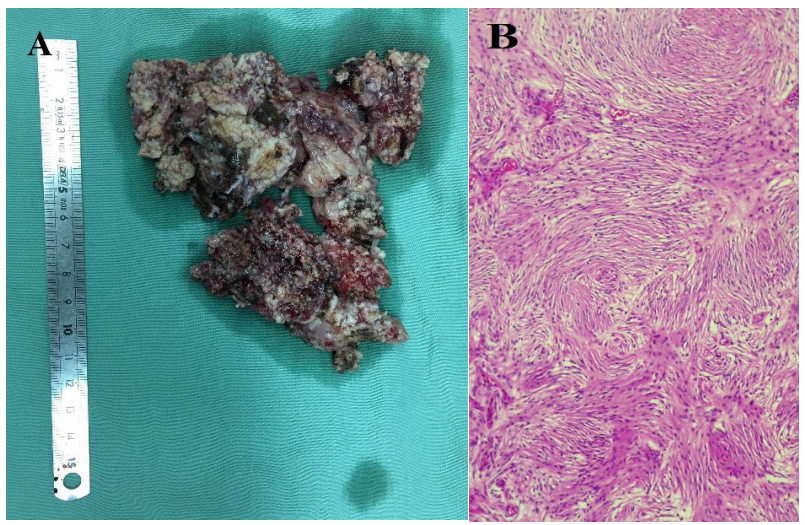

Figure 3. (A) Meningioma after surgery. (B). Meningioma WHO grade 1.

neuropsychological examination two months after the surgery. Since surgery, her symptoms were resolved and her quality of life has increased considerably.

\section{Discussion}

Meningioma is a slow-growing disease of the meninges. Ninety percent of meningiomas are benign and arise in the brain. Middle-age women are more than twice as likely as to experience meningioma than men. Many meningiomas develop during 30-70 years of age. ${ }^{5}$ This tumor occurs occasionally in children and adolescents ${ }^{6}$ and if not detected early and treated, it appears to develop into giant proportions and infiltrate surrounding tissues in early adulthood. This tumor is typically silent with no specific clinical manifestations. Studies have shown that during the luteal stage of the menstrual cycle and pregnancy, meningiomas change in size, and when estrogen agonist therapy is completed, they eventually worsen. ${ }^{1,7}$ Due to her age and early tumor recurrence, the treatment of the condition becomes more difficult. The World Health Organization grade I progesterone receptor, which is a strong prognostic predictor, is overwhelmingly optimistic. Chromosome 14 and 22 mutations, as well as molecular markers identified in meningothelial cell 
neoplastic changes, can be associated with elevated levels of estrogen receptor secretion. ${ }^{4,8}$ Meningioma has been associated with many family cancer predisposition syndromes that include gene-related syndromes with NF1, PTCH, CREBBP, VHL, PTEN, and CDKN2A. ${ }^{1,9}$ Susceptibility to ionizing radiation (IR) is actually the primary environmental risk factor for meningioma. ${ }^{1,9-11}$

Neurological symptoms, such as headaches, seizures, upper and lower limb fatigue, and sensory and visual disturbances are typically caused by meningioma. The patient's signs and symptoms are usually determined by the growth of the lesion and the site involved. ${ }^{12}$ The strong association between psychological conditions and the location of the lesions has been previously reported ${ }^{12,13}$ Depression is found predominantly in frontal convexity meningioma. Meningiomas of the bifrontal and sphenoid wing mainly present with mania or depression; and meningiomas with suprasellar lesions and temporal convexity often present with psychotic disorders. ${ }^{12}$ The seizures and tremors may be partly related to artery compression by the meningiomas at the left frontoparietal gyrus of a developing brain and partly due to intermittent disruption of the superior sagittal sinus and collateral or both, resulting in cerebral hypoxia and then seizures. The gold standard for preoperative examination is digital subtraction angiography and magnetic resonance angiography. ${ }^{14,15}$

Meningiomas have the "dural tail" on MRI. Ki-67 labeling index is used to measure the growing cells inside a tumor and MIB-1 monoclonal antibody is used to stain the Ki-67 antigen in histopathological estimates of the possible tumor growth. ${ }^{4,16}$ Experiments have shown elevated Ki-67 labeling indices in childhood and adolescent meningiomas but elevation does not predict relapse or prognosis., ${ }^{417}$ In comparison, positive GFAP cells have been associated with intrusive blood vessel-attached meningiomas. ${ }^{18}$ S-100 protein may help differentiate between meningiomas and schwannomas, while $90 \%$ of fibrous meningiomas are typically expressed as S-100 protein. ${ }^{3,19}$ Early diagnosis is really critical for our patient, since meningioma is typically benign and curable. As personality changes intensify, a delay in diagnosis can result in chronic disappointment for the family of the patient. Initially, our patient was diagnosed with suicidal symptoms, but then experienced some atypical symptoms that could not be clarified by any psychiatric diagnosis. This is an instance of a changeable clinical situation that needs early diagnosis and have a multi-discipline management approach subsequently.

A total tumor resection (Simpson I) is the medication of choice when a meningioma is in an available position to avoid recurrence and improve prognosis. Owing to their larger size, irregular location, and tight adhesion of the tumor to nerves and essential vessels, are challenging for surgical resections. Studies have shown that during operation, the cerebral veins that act as the normal drainage pathway, as well as those that can provide important collateral drainage, should be well-maintained and when the venous outflow is disrupted, venous infarction follows, leading to brain swelling, hemorrhage, and neuronal death. In general, slow and persistent venous occlusion is well-accepted, whereas acute occlusion can be disastrous. The maintenance of collateral drainage was further emphasized by researchers, since the sudden loss of superficial venous drainage due to destruction or thrombosis of the draining veins following surgery also contributes to neurological deficits..$^{20,21}$

\section{Conclusion}

Seeing giant meningiomas in a young adults is really rare. A matter of debate between neurosurgeons is still the cause of meningioma. Particularly for frontal lobe meningioma, clinical symptoms are atypical. If there is an irregular suspicion of psychological problems with the traditional "dural sign", MRI could be used. A total tumor resection to prevent recurrence and improve prognosis is the therapeutic option.

\section{Conflict of Interest}

The authors declare no conflict of interest.

\section{Authors Contribution}

Authors equally contributed the work.

\section{Funding/Support}

None.

\section{Ethical Statement}

This study approved by the ethical approval committee/Institutional Review Board of Hue University of Medicine and Pharmacy, No. 2380 / QD-DHYD, November 15, 2020.

\section{References}

1. Wiemels J, Wrensch M, Claus EB. Epidemiology and etiology of meningioma. J Neurooncol. 2010;99(3):307-14. doi: 10.1007/s11060-010-0386-3.

2. Mumoli N, Pulerà F, Vitale J, Camaiti A. Frontal lobe syndrome caused by a giant meningioma presenting as depression and bipolar disorder. Singapore Med J. 2013;54(8):e158-9. doi: 10.11622/smedj.2013160.

3. Yakhmi S, Sidhu BS, Kaur J, Kaur A. Diagnosis of frontal meningioma presenting with psychiatric symptoms. Indian J Psychiatry. 2015;57(1):91-3. doi: 10.4103/00195545.148534.

4. Prabhu VC, Perry EC, Melian E, Barton K, Guo R, Anderson DE. Intracranial meningiomas in individuals under the age of 30; analysis of risk factors, histopathology, and recurrence rate. Neuroscience Discovery. 2014;2(1):1-15. doi: 10.7243/20526946-2-1.

5. Gupta RK, Kumar R. Benign brain tumours and psychiatric morbidity: a 5-years retrospective data analysis. Aust $\mathrm{N}$ Z J Psychiatry. 2004;38(5):316-9. doi: 10.1080/j.14401614.2004.01357.x.

6. Caroli E, Russillo M, Ferrante L. Intracranial meningiomas in 
children: report of 27 new cases and critical analysis of 440 cases reported in the literature. J Child Neurol. 2006;21(1):316. doi: 10.1177/08830738060210010801.

7. Vadivelu S, Sharer L, Schulder M. Regression of multiple intracranial meningiomas after cessation of long-term progesterone agonist therapy. J Neurosurg. 2010;112(5):9204. doi: 10.3171/2009.8.jns09201.

8. Pravdenkova S, Al-Mefty O, Sawyer J, Husain M. Progesterone and estrogen receptors: opposing prognostic indicators in meningiomas. J Neurosurg. 2006;105(2):163-73. doi: 10.3171/jns.2006.105.2.163.

9. Simon M, Boström JP, Hartmann C. Molecular genetics of meningiomas: from basic research to potential clinical applications. Neurosurgery. 2007;60(5):787-98. doi: 10.1227/01.neu.0000255421.78431.ae.

10. Ron E, Modan B, Boice JD Jr, Alfandary E, Stovall M, Chetrit $A$, et al. Tumors of the brain and nervous system after radiotherapy in childhood. N Engl J Med. 1988;319(16):10339. doi: 10.1056/nejm198810203191601.

11. Sadetzki S, Flint-Richter P, Starinsky S, Novikov I, Lerman Y, Goldman B, et al. Genotyping of patients with sporadic and radiation-associated meningiomas. Cancer Epidemiol Biomarkers Prev. 2005;14(4):969-76. doi: 10.1158/10559965.epi-04-0366.

12. Bommakanti K, Gaddamanugu P, Alladi S, Purohit AK, Chadalawadi SK, Mekala S, et al. Pre-operative and postoperative psychiatric manifestations in patients with supratentorial meningiomas. Clin Neurol Neurosurg. 2016;147:24-9. doi: 10.1016/j.clineuro.2016.05.018.

13. Lampl Y, Barak Y, Achiron A, Sarova-Pinchas I. Intracranial meningiomas: correlation of peritumoral edema and psychiatric disturbances. Psychiatry Res. 1995;58(2):177-80. doi: 10.1016/0165-1781(95)02586-I.
14. d'Avella E, Volpin F, Manara R, Scienza R, Della Puppa A. Indocyanine green videoangiography (ICGV)-guided surgery of parasagittal meningiomas occluding the superior sagittal sinus (SSS). Acta Neurochir (Wien). 2013;155(3):415-20. doi: 10.1007/s00701-012-1617-5.

15. Richard SA, Shrestha SS, Zhang C, Fu W, Wang T, Cong W, et al. Successful treatment of a child with ruptured arteriovenous malformation using onyx embolization: a case report. Open J Mod Neurosurg. 2017;07(04):153-63. doi: 10.4236/ ojmn.2017.74016.

16. Abramovich CM, Prayson RA. MIB-1 labeling indices in benign, aggressive, and malignant meningiomas: a study of 90 tumors. Hum Pathol. 1998;29(12):1420-7. doi: 10.1016/ s0046-8177(98)90010-7.

17. Roser F, Samii M, Ostertag H, Bellinzona M. The Ki-67 proliferation antigen in meningiomas. Experience in 600 cases. Acta Neurochir (Wien). 2004;146(1):37-44. doi: 10.1007/s00701-003-0173-4.

18. Nakasu S, Fukami T, Jito J, Matsuda M. Microscopic anatomy of the brain-meningioma interface. Brain Tumor Pathol. 2005;22(2):53-7. doi: 10.1007/s10014-005-0187-0.

19. Hahn HP, Bundock EA, Hornick JL. Immunohistochemical staining for claudin-1 can help distinguish meningiomas from histologic mimics. Am J Clin Pathol. 2006;125(2):203-8. doi: 10.1309/g659-fvvb-mg7u-4rpq.

20. Andrews BT, Dujovny M, Mirchandani HG, Ausman Jl. Microsurgical anatomy of the venous drainage into the superior sagittal sinus. Neurosurgery. 1989;24(4):514-20. doi: 10.1227/00006123-198904000-00005.

21. Khu KJ, Ng I, Ng WH. The relationship between parasagittal and falcine meningiomas and the superficial cortical veins: a virtual reality study. Acta Neurochir (Wien). 2009;151(11):1459-64. doi: 10.1007/s00701-009-0379-1. 